JOURNAL OF SYNCHROTRON RADIATION

ISSN 1600-5775

Received 1 July 2016

Accepted 11 November 2016

Edited by P. A. Pianetta, SLAC National Accelerator Laboratory, USA

Keywords: instability; bunch by bunch; position.

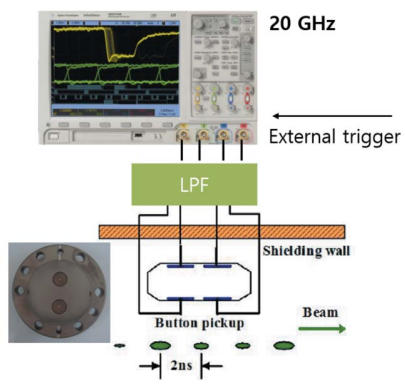

OPEN $\odot$ ACCESS

\section{Bunch-by-bunch position measurement and analysis at PLS-II}

\author{
Jaeyu Lee, ${ }^{a}$ M. H. Chun, ${ }^{a}$ G.-J. Kim, ${ }^{a}$ D.-C. Shin, ${ }^{a}$ D.-T. Kim ${ }^{a}$ and S. Shin ${ }^{a, b *}$ \\ aPohang Accelerator Laboratory, POSTECH, Pohang, Kyungbuk 37673, Republic of Korea, and ${ }^{\mathbf{b}}$ Advanced Photon \\ Source, Argonne National Laboratory, IL 60439, USA. *Correspondence e-mail: tlssh@postech.ac.kr
}

A bunch-by-bunch measurement system has been developed at Pohang Light Source II. The system consists of a four-channel button pick-up, $20 \mathrm{GHz}$ sampling oscilloscope and an $800 \mathrm{MHz}$ low-pass digital filter. Upon measuring a bunch-by-bunch spatio-temporal beam motion matrix over many turns, singularvalue decomposition analysis is used to reveal the dominant coupled-bunch modes. The system can diagnose injection oscillations due to kicker errors and the effect of resistive-wall impedance that gives rise to instability during operation.

\section{Introduction}

The upgraded Pohang Light Source II (PLS-II) (Shin et al., 2013) is now in full operation. As a result of the upgrade, the PLS beam energy increased from $2.5 \mathrm{GeV}$ to $3.0 \mathrm{GeV}$, and the stored beam current increased from $200 \mathrm{~mA}$ to $400 \mathrm{~mA}$. The emittance was improved from $18.9 \mathrm{~nm}$ at $2.5 \mathrm{GeV}$ to $5.8 \mathrm{~nm}$ at $3 \mathrm{GeV}$ while the PLS storage-ring tunnel structure remained unchanged. Top-up mode operation is used to stabilize the stored electron beam orbit and the synchrotron radiation flux.

During top-up operation (Emery, 2001), the beam injection excites an oscillation of the stored beam. The oscillation increases the effective stored beam emittance and modulates the photon beam intensity. For example, $1-8 \%$ systematic dips in the photon beam flux at each beamline occurs during top-up injection in PLS-II (Hwang et al., 2014). Furthermore, storagering-based light sources will further evolve toward ultra-low emittance. In these light sources, the lifetime of the stored electron beam will be short and the frequency of beam injection by top-up will increase. Therefore, to achieve high performance of top-up injection for user experiments in future low-emittance rings, the stored beam oscillation must be suppressed during top-up injection.

Beam dynamics phenomena due to bunch-by-bunch $(\mathrm{BbB})$ motion have important effects in a storage ring. For instance, partial or entire beam loss occurs occasionally at PLS-II. Through fill pattern measurements, we have identified coupled bunch instability as one of the reasons for this. Direct measurements of the beam position related to different dynamical mechanisms provide useful information that can be used to guide accelerator optimization. At PLS-II, a $20 \mathrm{GHz}$ sampling oscilloscope synchronized with injection events (or triggered by beam loss signals) is used to measure $\mathrm{BbB}$ motion. Based on the measured data, principal component analysis was performed to provide insight into dynamic phenomena such as coupled bunch instabilities and beam oscillations due to injection kicker mismatch. 
In this paper we describe the $\mathrm{BbB}$ position measurement system in PLS-II. $§ 2$ presents the system layout and data processing for the $\mathrm{BbB}$ beam position monitor (BPM). $\$ 3$ describes the analysis of injection transients using the BbB BPM. Analysis of the instabilities is described in $\S 4$, and $\S 5$ presents conclusions.

\section{System layout and data processing}

The PLS-II BbB measurement system (Fig. 1) consists of a BPM signal pick-up, $750 \mathrm{MHz}$ analogue low-pass filter (LPF) and a $20 \mathrm{GHz}$ sampling oscilloscope. The pick-up is located at cell 12 in the PLS-II storage ring. A typical PLS-II BPM (Hong et al., 2011) is used. The BPM consists of an octagonal vacuum chamber and four pick-up electrodes. The vacuum chamber has a horizontal aperture of $66 \mathrm{~mm}$ and a vertical aperture of $22 \mathrm{~mm}$. The horizontal separation between two pick-up electrodes, which is mainly defined by sensitivity, is $15 \mathrm{~mm}$. The pick-up electrode for the BbB BPM consists of a central conductor with a button electrode, an outer conductor with a SubMiniature version A-type feedthrough, and a ceramic insulator. In time-domain simulations, the generated output signal is completely damped within $0.5 \mathrm{~ns}$ (Fig. $2 a$ ). The time gap between bunches is $\sim 2 \mathrm{~ns}$ and the harmonic number is 470 .

The frequency content of the signal, i.e. the Fourier transform of Fig. 2(a), is broadband. The BbB motion of interest is at frequencies $f<500 \mathrm{MHz}$ with the high-frequency signal in the time domain sensitive to timing jitter, so the BPM signal at $f<500 \mathrm{MHz}$ is obtained by using the low-pass filter SBLP-467 (Fig. 2b). An Agilent oscilloscope records the broadened signal at a $20 \mathrm{GHz}$ sampling rate; unfortunately, the recorded data contained noise with $f>500 \mathrm{MHz}$ (Fig. 2c). Therefore, a digital FIR filter with 815 th order and $800 \mathrm{MHz}$ pass frequency was used to remove highfrequency noise signals (Fig. $2 d$ ).

The sampling rate of the oscilloscope was not an integer multiple of the RF that is obtained from the discrete Fourier transformation of the raw waveform, so cubic spline interpolation was used to obtain the bunch positions at adjusted signal phases. The $\mathrm{BbB}$ position time series was rearranged and transformed into turn-by-turn (TbT) position information for each bunch so that a bunch position matrix was assembled for beam dynamics analysis.

\section{Diagnosis of injection transient}

In top-up mode operation, a small amount of beam current is injected into the stored beam to keep the total beam current almost constant. During top-up

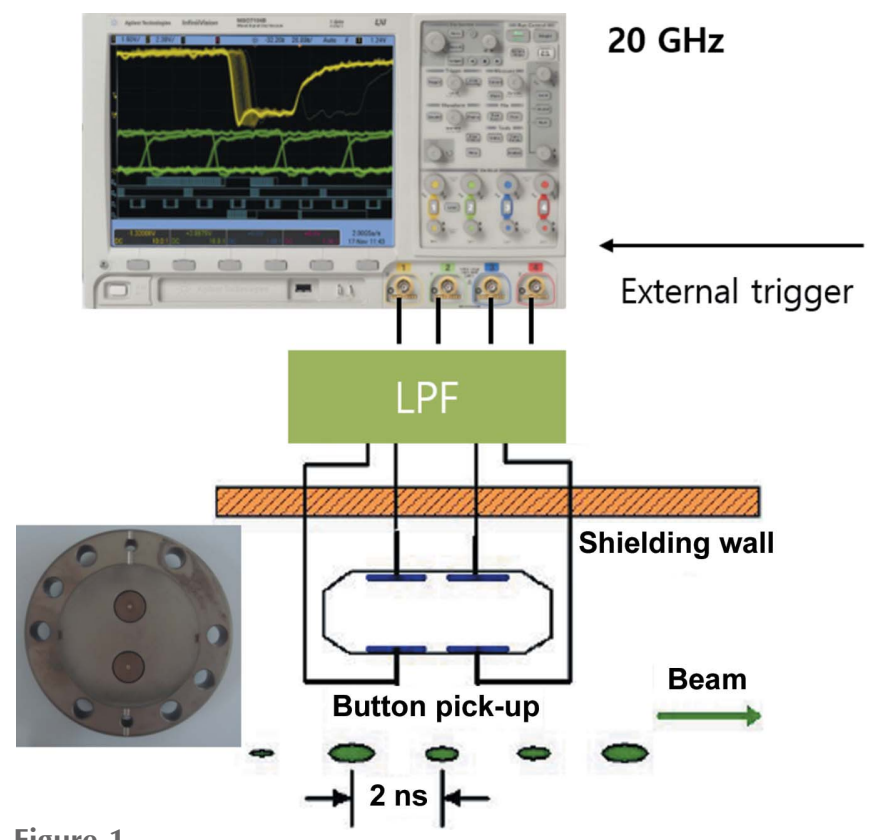

Figure 1

Layout of the BbB BPM system. Analogue low-pass filter and $20 \mathrm{GHz}$ sampling oscilloscope translate the analogue pick-up signal to raw digital data. An external trigger from the timing system is synchronized with the injection event.

injection, a four-kicker system deflects the position of a stored bunch near the injected one, and then moves it back to the original orbit with the nearly injected bunch. Unfortunately, an oscillation in the stored beam will cause a perturbation in beamline experiments due to rotation of the bump magnets, the leakage field from the septum magnet, and the differences

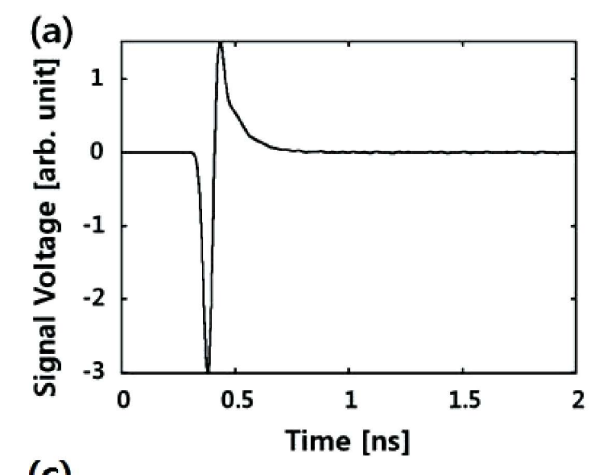

(c)

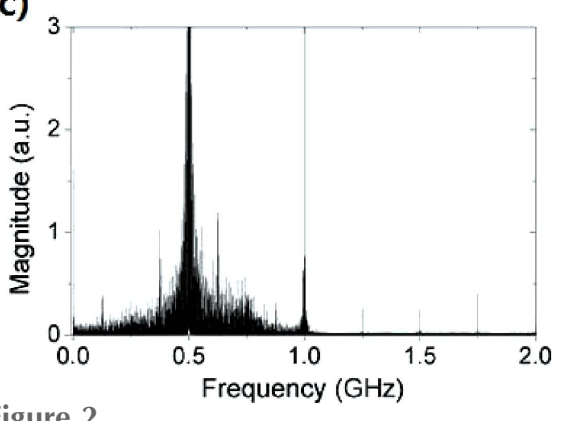

Figure 2

Analogue data processing. (a) Simulated pick-up signal in the time domain. (b) Measured pick-up signal after and before the analogue LPF. Digital data processing. (c) FFT of raw data. (d) Raw data and filtered data with $800 \mathrm{MHz}$ LPF. (b)

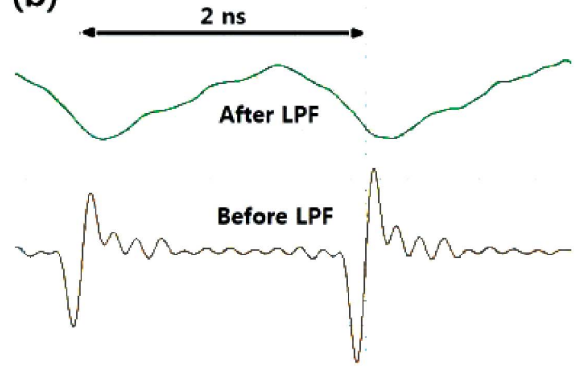

(d)

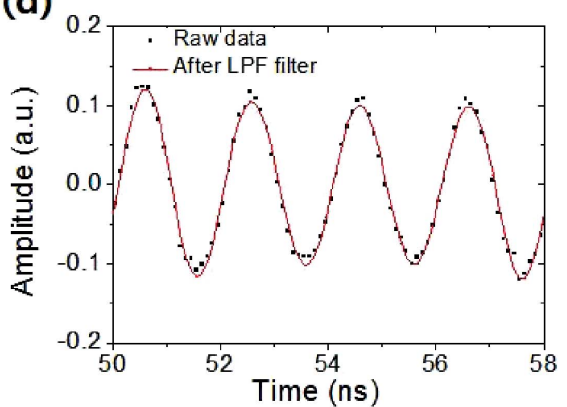



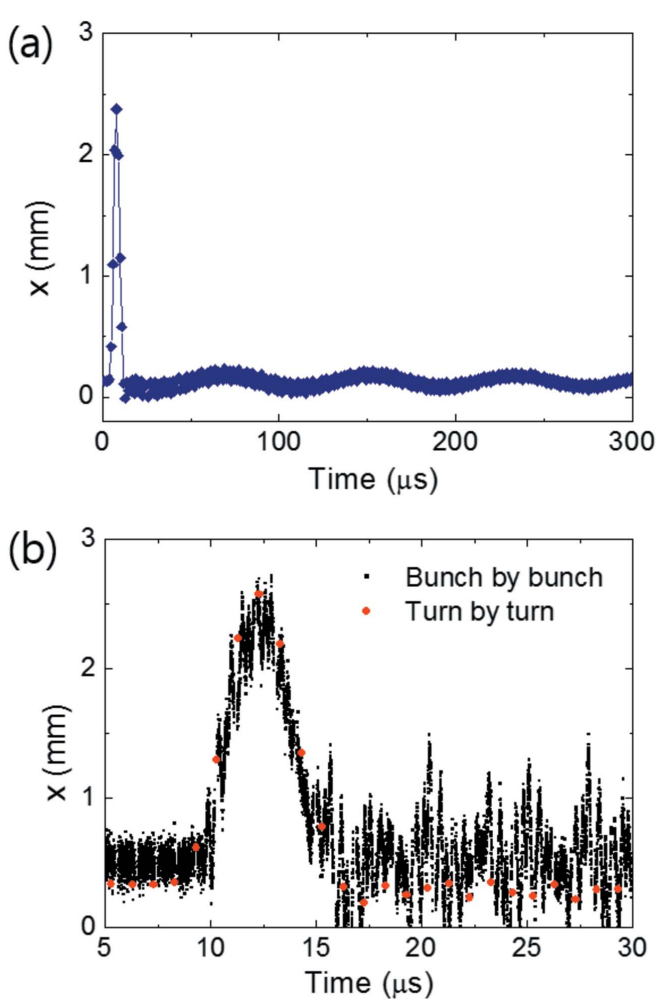

Figure 3

Injection transient analysis using turn by turn (TbT) BPM and bunch-bybunch (BbB) BPM. (a) TbT BPM analysis on the horizontal oscillation during $300 \mu$ s after kicker turn-on. (b) Comparison between TbT BPM and BbB BPM data.

among the magnetic fields of the four kicker magnets. The stored beam has a bumped orbit of $7 \mu$ s after the kicker magnet turn-on, and the orbit continues to oscillate with a period of $\sim 100 \mu \mathrm{s}$ (Fig. 3a). The oscillation of the stored beam affects the photon position and flux stability in beamline experiments. Therefore, the source of stored beam oscillation must be determined and suppressed.

To analyse the injection transient motion, we compared the TbT position data and the BbB position measurement in the horizontal plane during the first $20 \mu$ s after the kicker magnet was turned on (Fig. 3b). The BbB position data implicitly show the excitation of the beam oscillation due to waveform errors of the kickers, and the systematic error due to field asymmetry of the kicker.

To explore the physical meaning of the $\mathrm{BbB}$ position data, singular-value decomposition (SVD) analysis was applied to the matrix $B$ where rows represent turns and columns represent bunch index. SVD yields a spatialtemporal modal analysis of beam

Figure 4 motion by effectively accomplishing statistical principal component analysis. Mathematically, SVD of the matrix $B$ yields (Wang, 2003)

$$
B=U S V^{T}=\sum_{i=1}^{d} \sigma_{i} u_{i} v_{i}^{T}
$$

where $U_{P \times P}=\left[u_{1}, \ldots, u_{P}\right]$ and $V_{M \times M}=\left[v_{1}, \ldots, v_{M}\right]$ are orthonormal matrices, $S_{P \times M}$ is a diagonal matrix with nonnegative $\sigma_{i}$ along the diagonal in decreasing order, $d=\operatorname{rank}(B)$ is the number of non-zero singular values, and vectors $u_{i}$ and $v_{i}$ are the $i$ th left and right eigenvectors, respectively. Each set of $\left\{u_{i}, v_{i}\right\}$ defines a spatio-temporal mode, where $u_{i}$ gives the temporal variation (turns) and $v_{i}$ gives the spatial variation (bunch position). The singular values reveal the system dimensionality and relative magnitudes, and each set of singular vectors $U_{P \times P}$ and $V_{M \times M}$ forms an orthonormal basis for the time and bunch position spaces of the matrix.

To analyze injection transients we performed SVD analysis for the matrix $B$ with 1000 samples each for the 430 bunches. Here, 1000 samples correspond to $1 \mathrm{~ms}$ of data. The diagonal elements of the singular value matrix $S$ provide an estimate of the modes. The singular-value spectrum has discrete floors, and a few modes are separable from the floors (Fig. $4 a$ ). The first singular value of the matrix is predominantly large. It indicates that there is a dominant motion of the matrix. Figs. 4(b) and 4(c) show the first temporal eigenvector from matrix $B$ and its frequency component, respectively. The first temporal eigenvector (Fig. $4 b$ ) of $B$ clearly shows typical horizontal beam motion that consists of a large orbit shift while the kicker is on, and energy oscillation and betatron
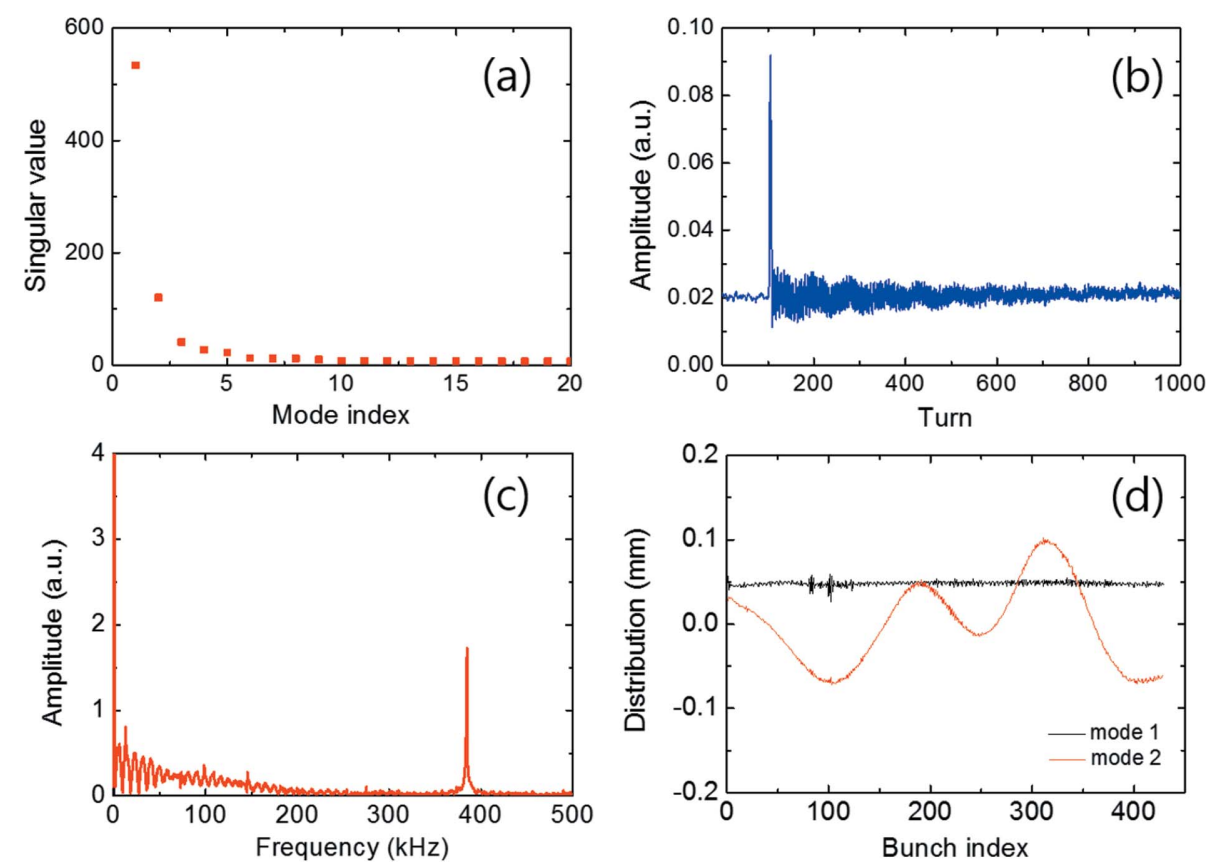

SVD analysis of the horizontal injection transient using BbB BPM. (a) Singular values of the separated modes. The first principal mode is dominant and noise is negligible. $(b)$ The oscillation amplitude over turns of the first principal mode. (c) FFT of the oscillation amplitude. (d) Distributions of the first two principal modes along the bunches. 
oscillation after the kicker is turned off. The frequency component of the first temporal eigenvector shows betatron tune and synchrotron tune (Fig. 4c). Based on the singular values, only two major modes with relatively large singular values were present (Fig. 4d). The first mode is uniform along the bunch index and the second mode shows oscillation along the bunch index. Therefore, the singular value of the first mode is considered as the amplitude of the injection bump. The betatron damping associated with the singular value of the second mode is considered as oscillation that the kicker waveform error causes in amplitude along the bunch. As a result, a $3 \mathrm{MHz}$ waveform error is assumed in the kicker waveform from the amplitude modulation along the bunch in the second mode.

\section{Diagnosis of instabilities and beam dump}

An intermediate storage ring like the PLS-II with a beam energy of $3 \mathrm{GeV}$ can deliver high photon energies. In-vacuum undulators (Kim et al., 2016) with a period length of $20 \mathrm{~mm}$ and a peak field of $0.97 \mathrm{~T}$ are used in the PLS-II ring to produce such X-rays in the fundamental or higher harmonics. Twelve narrow-gap in-vacuum undulators are presently in operation; they unfortunately produce coupled bunch instabilities by resistive wall impedance and thereby limit the stored beam current. Therefore, a BbB feedback system is needed to suppress coupled bunch instabilities (Lee et al., 2014).

A broadband feedback system for transverse betatron oscillations or longitudinal synchrotron oscillations is an effective device for suppressing beam instabilities that limit the beam current or the quality of the stored beam. Moreover, the BbB feedback system can damp the beam oscillations excited by injection perturbations that are harmful to user experiments. The BbB feedback system detects transverse or longitudinal positions of a beam, processes the position data to calculate the actuator signal, and kicks the beam to damp the oscillations. To develop a standard to upgrade the transverse feedback system (TFS) to improve precision, the instability phenomenon during high-current operation must be understood.

If the TFS is turned off under normal operation conditions (360 mA with 400 bunches), beam loss occurs (Fig. 5a). The dynamics of coupled bunch instability is investigated using a $\mathrm{BbB}$ position measurement. Of the total of $470 \mathrm{RF}$ buckets, 400 are full and 70 are empty (ion clearing gap). Bunches in the tail part are lost. Compared with horizontal motion, vertical bunch motion has large oscillations (Fig. $5 b$ ); due to a fractional vertical tune of 9.145 , the oscillation peak is repeated every seven turns. This implies strong vertical betatron oscillation. In addition to strong vertical betatron oscillation, the bunch train shows obvious betatron phasing. The beam seems to be under the influence of the first coupled bunch mode excited by the resistive wall impedance. Compared with the first bunch oscillation, the 350th bunch oscillation is large and causes beam loss (Fig. 5).
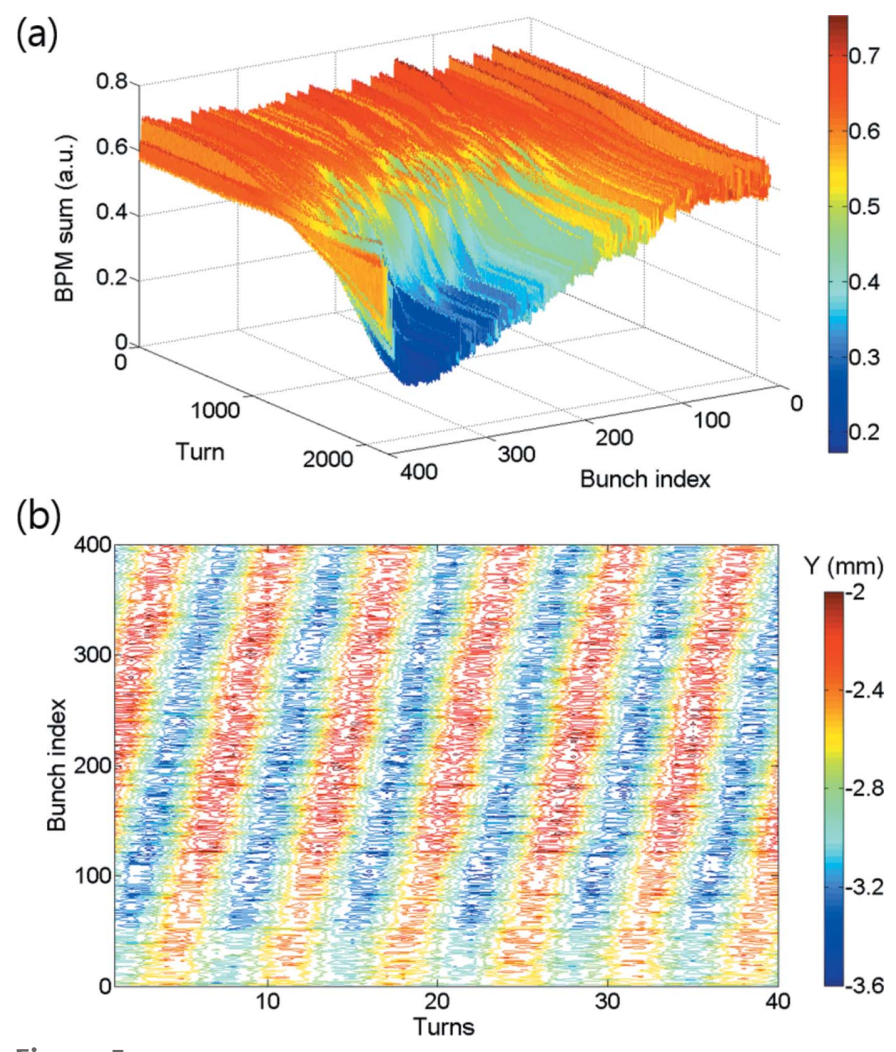

Figure 5

BbB BPM analysis during the beam loss from the coupled bunch instability. (a) BbB BPM sum during the last $2 \mathrm{~ms}$ before the beam loss. (b) $\mathrm{BbB}$ vertical oscillation before the beam loss.

A general equation for coupled bunch motion of the beam considering a wake function over the entire circumference is

$$
y_{n}^{\mu} \propto \exp \left(2 \pi i \frac{\mu n}{M}\right) \exp \left(-i \Omega_{\mu} t\right)
$$

where mode number $\mu$ gives the betatron phase advance between one bunch and the next, and mode frequency $\Omega_{\mu}$ describes the frequency of oscillations as a bunch moves around the ring. This expression describes the behavior of a mode that consists of a regular pattern of transverse bunch positions that oscillates at a particular frequency; the imaginary part gives the growth rate of the corresponding mode. For the PLS-II normal operation $(360 \mathrm{~mA}$ beam current), inserting a total of $10 \mathrm{~m}$ of $6 \mathrm{~mm}$ gap in-vacuum undulators (copper plate) and considering the $11 \mathrm{~mm}$ gap in the $270 \mathrm{~m}$-long aluminium pipe, the estimated growth time is $\sim 2 \mathrm{~ms}$.

The SVD analysis of vertical bunch motion seen in Fig. 5(b) can be used to quantify beam motion under the condition of beam instability (Fig. 6). In the SVD analysis, the first two singular values are dominant, and others are much lower floors of singular values. The first temporal and spatial eigenvectors that corresponds to $\mu=0$ are shown in Figs. 6(a) and Fig. $6(b)$, respectively; together they describe coherent betatron oscillation (no phase advance between bunches). The estimated growth time was $0.5 \mathrm{~ms}$ (Fig. $6 a$ ). In practice, many components in addition to the narrow-gap in-vacuum undu- 

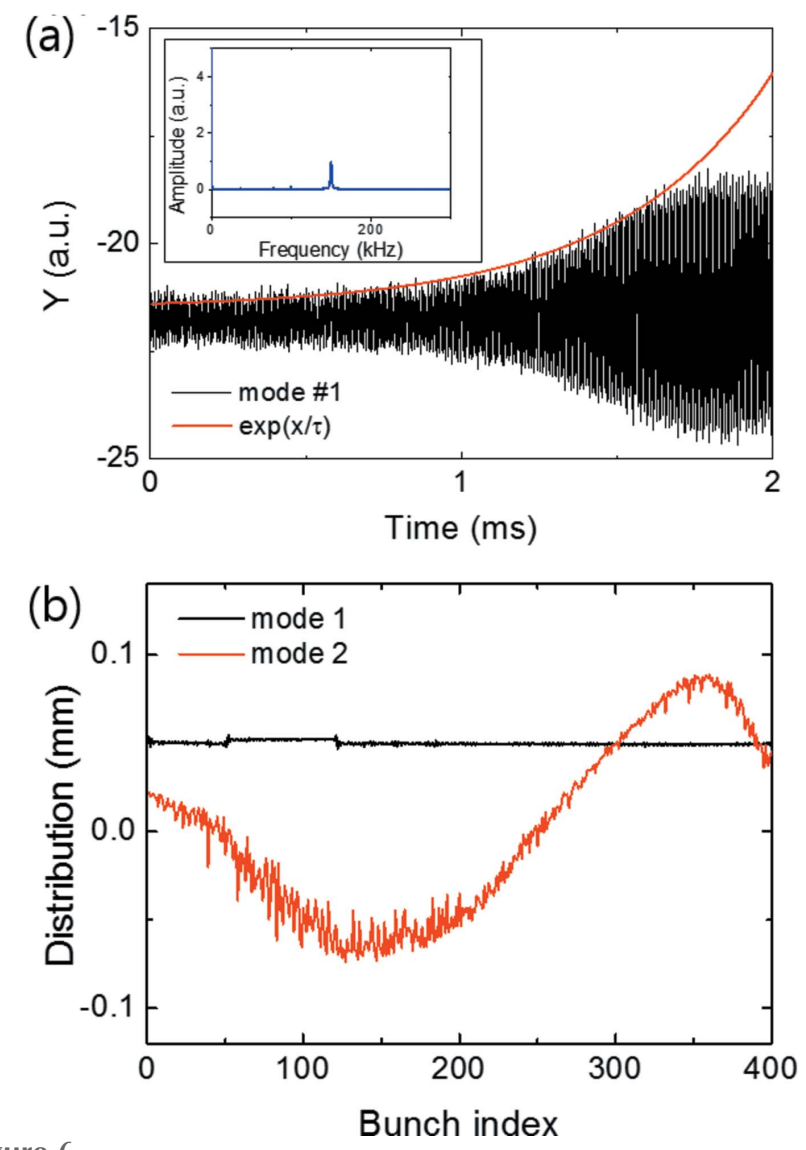

Figure 6

(a) Vertical position of the 350th bunch during coupled bunch instability and its exponential fitting function. (b) Distribution of the first two principal modes along the bunches obtained using the SVD algorithm.

lator contribute to the ring impedance (e.g. kicker magnets, vacuum components, cavity-like structures). These components reduce the growth time. The second spatial eigenvector in Fig. $6(b)$ is the first coupled bunch mode excited by the resistive wall impedance.

\section{Conclusion}

Turn-by-turn measurements of the transverse beam position in storage rings typically have limits for the investigation of injection transients, and coupled bunch instabilities. We designed and implemented a wideband bunch-by-bunch transverse position measurement system at the PLS-II. The effects of field balance error and waveform error in the injection kickers was revealed through bunch-by-bunch measurements of the injection transient. Beam loss processes from resistive wall impedance were also observed and investigated. The first coupled bunch mode excited by resistive wall impedance is dominant during beam loss and the growth time of coherent betatron oscillation was measured to be $0.5 \mathrm{~ms}$, which is less than the $2 \mathrm{~ms}$ calculated value.

\section{Acknowledgements}

We thank T. Nakamura and R. Nagaoka for useful discussions. This research was supported by the Converging Research Center Program through the Ministry of Science, ICT and Future Planning, Korea (NRF-2014M3C1A8048817), and the Basic Science Research Program through the National Research Foundation of Korea (NRF-2015R1D1A1A01060049).

\section{References}

Emery, L. (2001). Proceedings of the 19th IEEE Particle Accelerator Conference (PAC'01), Chicago, IL, USA, 18-22 June 2001, p. 2599. Hong, J., Lee, S., Ko, I. S., Kim, C., Kim, D. T., Park, H. J., Lee, E. H., Lee, J. W., Kim, S., Park, Y. \& Par, S.-J. (2011). J. Kor. Phys. Soc. $\mathbf{5 9}, 594$.

Hwang, I., Huang, J. Y., Kim, M., Lee, B.-J., Kim, C., Choi, J.-Y., Kim, M.-H., Lee, H. S., Moon, D., Lee, E. H., Kim, D.-E., Nam, S. H., Shin, S. \& Cho, M. (2014). Rev. Sci. Instrum. 85, 055113.

Kim, D.-E., Lee, H.-H., Park, K.-H., Seo, H.-S., Ha, T., Jeong, Y.-G., Han, H.-S., Lee, W. W., Huang, J.-Y., Nam, S., Kim, K.-R. \& Shin, S. (2016). J. Korean Phys. Soc. 69, 903-908.

Lee, E. H., Kim, D. T., Huang, J. Y., Shin, S., Nakamura, T. \& Kobayashi, K. (2014). Rev. Sci. Instrum. 85, 125102.

Shin, S., Kwon, S., Kim, D., Kim, D., Kim, M., Kim, S., Kim, S., Kim, J., Kim, C., Park, B., Park, S., Park, S., Park, E., Son, Y., Yoon, J., Lee, B., Lee, E., Lee, J., Lee, H., Joo, Y., Choi, J., Ha, T., Hwang, W., Hwang, I., Lee, J., Oh, B., Lee, C., Lee, H., Kim, J., Hwang, J. Y., Nam, S. H. \& Cho, M. (2013). J. Instrum. 8, P01019.

Wang, C. X. (2003). Proceedings of the 2003 IEEE Particle Accelerator Conference (PAC 03), Portland, OR, USA, 12-16 May 2003, pp 3410-3412. 